\title{
Caged for protection
}

To metastasize, tumour cells must traverse the tight pores of the extracellular matrix (ECM) by either degrading the surrounding ECM or constricting their shape. If using the latter mechanism, the tumour cell must deform its stiffest component, the nucleus, sufficiently to squeeze through the small spaces, which can lead to nuclear envelope rupture and associated DNA damage. Skau and colleagues now describe a protective cytoskeletal structure that forms around the nucleus and ensures that its translocation is not a rate-limiting step in confined migration during metastasis.

The authors focused on a possible role for the actin nucleation factor formin 2 (FMN2) in cell migration, as FMN2 had previously been shown to induce nuclear actin filament assembly. On 2D surfaces, FMN2 localized to a subset of actin bundles and focal adhesions - different in composition and activity - positioned beneath the nucleus of primary mouse embryonic fibroblasts (MEFs) and mouse B16-F10 melanoma cells. These perinuclear actin bundles and focal adhesions contained myosin $2 \mathrm{~B}$ and could deform and move with the nucleus. Knockdown of FMN2 with short interfering RNA (siRNA) in MEFs (FMN2-KD) disrupted the cytoskeletal structure and mislocalized myosin $2 \mathrm{~B}$ to the cell periphery when compared with mock-transfected controls, confirming that FMN2 is required to generate the perinuclear actin and focal adhesion system. Furthermore, FMN2-KD cells migrating on top of ECM in vitro displayed an increase in nuclear lobularity and nuclear mislocalization from the cell centre, indicating that FMN2 controls both nuclear shape and position during migration in 2D.

Moving to 3D microenvironments, Skau et al. showed that FMN2 formed a cup-like shape around the rear of the nucleus of cells as they migrated through collagen ECM, and FMN2-KD, as in 2D, resulted in loss of actin bundles and focal adhesions from the perinuclear region accompanied by a loss of cell polarization. Interestingly, survival of FMN2-KD cells inversely correlated with collagen concentration. As this decreased viability was not observed in $2 \mathrm{D}$, the authors hypothesized that confinement during invasion may be responsible for inducing cell death in the absence of FMN2.

In line with this theory, 3D invasion assays revealed that migration of FMN2-KD cells through different pore sizes was impaired compared with control cells. The few FMN-KD cells that could migrate though the pores had an increased number of DNA double-strand break (DSB) repair sites, quantified by foci of phosphorylated histone $\mathrm{H} 2 \mathrm{AX}$ and 53BP1. Moreover, the amount of DNA damage increased as pore size decreased, directly showing that DNA damage was a function of physical constraint. Use of specific kinase inhibitors demonstrated that the DNA damage response in these invading cells was activated by an ataxia telangiectasia mutated (ATM) and not an ataxia telangiectasia and Rad3-related protein (ATR)-dependent signalling pathway. Further experiments showed that the actin polymerization activity of FMN2 was necessary for protecting the nucleus and its contents.

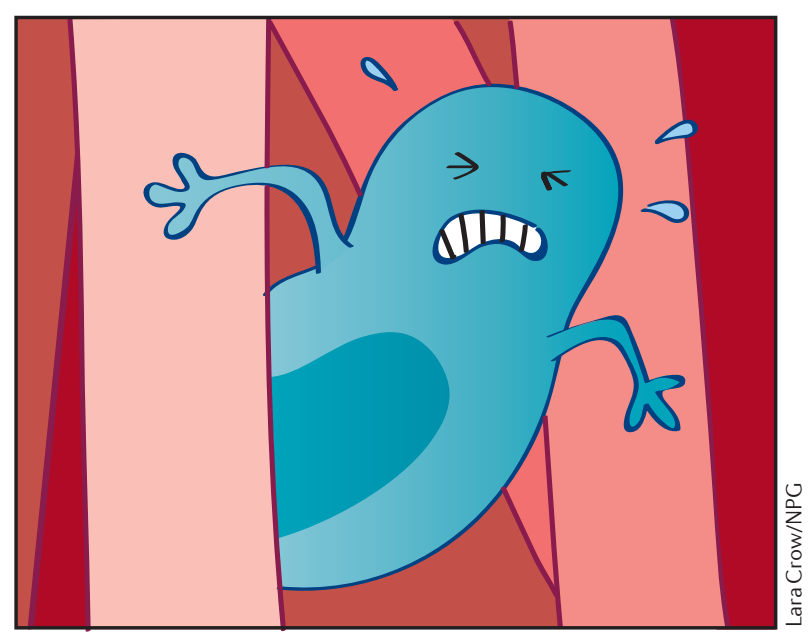

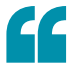

FMN2

promoted cell

survival during confined migration

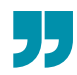

In support of a protective function for the FMN2-mediated perinuclear actin and focal adhesion system from mechanically induced damage, FMN2-KD cells confined within microchannels with constrictions of varying widths underwent extensive and irreversible loss of nuclear envelope integrity in the narrow sections, whereas control cells could migrate through them with only transient rupture of the nuclear envelope. Given that FMN2 promoted cell survival during confined migration, the authors reasoned that FMN2 might also be important during metastasis of cancer cells. Indeed, Skau et al. found that B16-F10 cells lacking FMN2 had reduced extravasation into the lungs with a striking $91 \%$ decrease in metastasis formation following intravenous injection into mice.

Taken together, these results suggest that FMN2 upregulation in cancer cells might promote metastatic phenotypes and that targeting FMN2 could be a potential anti-metastatic strategy.

Anna Dart

ORIGINAL ARTICLE Skau, C. T. et al. FMN2 make perinuclear actin to protect nuclei during confined migration and promote metastasis. Cell http://dx.doi.org/10.1016/j.cell.2016.10.023 (2016) 